\title{
STATISTICAL GEOMETRY: A TOOL FOR PATTERN ANALYSIS ${ }^{1}$
}

BY ULF GRENANDER

0. Summary. This paper deals with the analysis and recognition of two-dimensional set patterns deformed by certain deformation mechanisms. The emphasis is on exploiting the form of the image algebra and the behavior of the deformations. In this way higher recognition efficiency is obtained by using structure-preserving restoration.

1. The structure of patterns. Patterns can be described through the following general formalism. They are generated from primitives called signs and denoted by $s, s \in \mathscr{S}$. Finite vectors $c=\left(c_{1}, c_{2}, \ldots, c_{m}\right) \in \mathscr{C}$ are called configurations and in the set $\mathscr{C}$ of all legal configurations an equivalence relation $R$ gives rise to equivalence classes $I$ called images. A set of rules $\mathscr{R}$ determines what configurations are legal. The set $\mathscr{T}$ of resulting images is called the image algebra. Expressing configurations just as vectors is sufficient when we have just one binary operation between signs. If several binary operations are used a more expressive notation is needed; see below. The images $I$ are the objects that can be observed (under ideal conditions) and, depending upon the way they have been generated, they are grouped into pattern classes $\mathscr{P}_{\alpha}$ forming a family $\mathscr{P}$ of patterns. The reader is referred to Grenander (1970) for more details.

Under actual conditions the images cannot be observed exactly. Instead, a deformation mechanism $\mathscr{D}$ maps the set $\mathscr{T}$ of pure images into a set $\mathscr{T}^{\mathscr{D}}$ of deformed images. The purpose of pattern analysis is to describe the generation of $\mathscr{T}$, the mapping into $\mathscr{T}^{\mathscr{D}}$, and to design algorithms for the analysis and recognition of I given $I^{\mathscr{D}}$ or, at least, the partial restoration of $I$. Finally this will be used for classification into the pattern classes $\mathscr{P}_{\alpha}$.

The main difficulty is usually caused by the way that $\mathscr{D}$ obscures the view of the pure image $I$. Often the deformation mechanism $\mathscr{D}$ is not even discussed explicitly, but only assumed vaguely when the recognition algorithm is suggested. In this paper we shall show how to take $\mathscr{D}$ into account for the special case of two-dimensional set patterns and for a

An address delivered before the Providence meeting of the Society on October 28, 1972 by invitation of the Committee to Select Hour Speakers for Eastern Sectional Meetings; received by the editors January 4, 1973 and, in revised form, February 14, 1973.

AMS (MOS) subject classifications (1970). Primary 68A45.

Key words and phrases. Pattern analysis, deformations, image algebras.

${ }^{1}$ Supported by NSF grant GJ-31107X. 
certain choice of $\mathscr{D}$. For other $\mathscr{D}$ 's of background deformation type, see Grenander (1970) and Kowalczyk (1972).

In the present case the signs are certain convex, closed subsets of the plane. Starting from a set Proto of prototypes we transform them by the action of a group $G$ of transformations $g$ of the plane onto itself. $G$ is made up of three groups: Trans $=$ translations, Rot $=$ rotations, and Scale $=$ scale changes of $R^{2}$. We may also let $G$ be some subgroup of these. This means that

$$
\mathscr{S}=G \cdot \text { Proto }
$$

Lebesgue area will be denoted by $m$.

Signs will be combined by unions and intersections and we shall also allow complements to be taken. The relation $R$ will be used to identify the resulting sets. If two configurations $A$ and $B$ are equivalent modulo $R$, $A \equiv B(\bmod R)$, this means that the two resulting sets are identical, not that they have been generated in the same way. If $A$ is included in $B$, for example, then the images $A$ and $A \cap B$ are equivalent although the configurations are different.

In general, if $B$ is a Boolean function in $k$ variables and if $s_{1}, s_{2}, \ldots$, $s_{k} \in \mathscr{C}$ then we can speak of the image

$$
I=B\left(s_{1}, s_{2}, \ldots, s_{k}\right),
$$

assuming of course that the constraint $\mathscr{R}$, to be defined below, is not violated.

Let us mention a few examples that will be used repeatedly in the following sections. If Proto consists only of the single prototype $=C=$ unit circle and if $G=$ Trans $\times$ Scale, we get, for example, the disjunctive images in Figure 1.1(d) and the conjunctive images in Figure 1.1(a), (b), (c). With the prototype $S_{i}=$ squares, $i=1,2$, we can generate the conjunctive image in Figure 1.1(e). Many other shapes can be obtained from the same prototypes but it should be observed that only small values of $k$ are of interest in (1.2) since otherwise the flexibility is so large that almost any shape can be well approximated by the resulting images and the algebra has little influence in governing the shape of the images.

We shall treat three different deformation mechanisms that are closely related.

$\mathscr{D} 1 . I^{\mathscr{D}}$ is a sample of $n$ points drawn at random from a uniform distribution over $I$.

$\mathscr{D} 2 . I^{\mathscr{D}}$ is the realization of a Poisson process with density $\lambda$ over $I$ (no points outside of $I$ ).

$\mathscr{D} 3 . I^{\mathscr{D}}$ is the realization of a Poisson process with density $\lambda_{1}$ inside of $I$ and $\lambda_{2}$ outside of $I$, with $\lambda_{1}>\lambda_{2}$. 
(a)

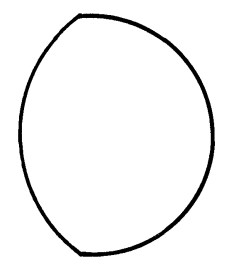

$\left(g_{1} C\right) \cap\left(g_{2} C\right)$ (b)

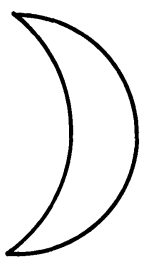

$\left(\sim g_{1} C\right) \cap g_{2} C$

(d)

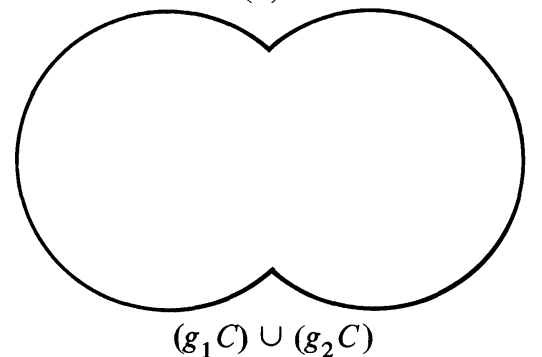

(c)

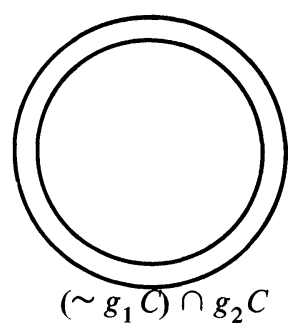

(e)

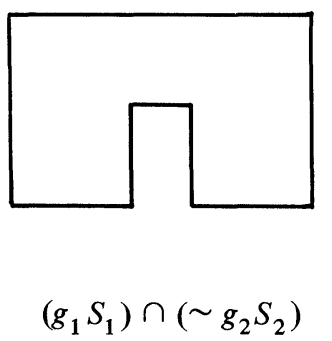

FIGURE 1.1

It is well known that $\mathscr{D} 1$ can be considered a conditional form of $\mathscr{D} 2$, fixing the number of points, so that they can be treated in almost the same way. Also, $\mathscr{D} 2$ is a special case of $\mathscr{D} 3$.

In the following sections we shall devise methods for restoring $I$ through some set $I^{*}$ calculated from the observed point cluster $I^{\mathscr{D}}$. Of course we would like $I^{*}$ to be close, in some sense, to the pure image $I$.

We shall approach our task from two directions. First we shall use the special properties of the image algebra and in particular exploit the behavior of the boundary $\partial I$ of $I$. The deformed image $I^{\mathscr{D}}$ does not have any nontrivial boundary, strictly speaking. Nevertheless, we shall use "boundary" properties of $I$ to estimate $\partial I$ and then go on to $I$ itself. To do this we shall introduce a number of boundary statistics.

In later sections we shall not use the boundary properties of $I^{\mathscr{D}}$ as directly as mentioned, although they will still appear implicitly. Finally, we shall design methods that do not use at all the algebra underlying the image algebra $\mathscr{T}$; it can be expected that such very general algorithms are less efficient in restoring images known to belong to $\mathscr{T}$ than the ones that take advantage of the algebraic structure. This will lead us to study structure-preserving methods of pattern analysis. We shall begin this in the present paper; the study will be continued in Grenander and Lavin (1973). 
If $I^{\mathscr{D}}=\left\{z_{1}, z_{2}, \ldots, z_{n}\right\}$, where each $z_{i}$ is a point in the plane, the likelihood function (whose argument is the unknown image $I$ ) is

$$
L_{1}(I)=\left\{\begin{array}{cl}
{[m(I)]^{-n}} & \text { if } I^{\mathscr{D}} \subset I, \\
0 & \text { else, }
\end{array}\right.
$$

so that the likelihood estimator $I^{*}$ is the one that solves the minimum problem

$$
m(I)=\min _{I^{\mathscr{Q}} \subset I} .
$$

This is for $\mathscr{D} 1$. For $\mathscr{D} 2$ we get instead of (1.3)

$$
L_{2}(I)=\lambda_{1}^{n} \exp \left(-m(I) \lambda_{1}\right)
$$

if we treat the $z$ 's as unordered; otherwise the expression should be divided by $n$ !. The ML-estimator is still given by (1.4). If $\mathscr{D} 3$ is the deformation mechanism we get instead

$$
L_{3}(I)=\lambda_{1}^{n_{1}} \exp \left(-\lambda_{1} m(I)\right) \lambda_{2}^{n_{2}} \exp \left(-\lambda_{2} m\left(I^{c}\right)\right)
$$

where $I^{c}$ stands for the complement of $I, n_{1}$ is the number of $z$ 's inside $I$ and $n_{2}$, inside $I^{c}$. The ML-estimator is obtained by solving the minimum problem

$$
\left(\lambda_{1}-\lambda_{2}\right) m(I)-n \log \left(\lambda_{1} / \lambda_{2}\right)=\min _{I \in \mathscr{T}}
$$

Once we possess efficient means of restoring and recognizing images the final problem of identifying pattern classes is a good deal closer to its solution (see Grenander (1970)).

By statistical geometry we mean the study of how to restore pure geometric objects when we can only observe deformed versions of them.

The problem studied in this paper concerns the identification of objects in a given space. Another area of statistical geometry is devoted to the identification of intrinsic properties of the space itself. Such properties could be metric, connectivity, and neighborhood relations. Problems of this nature will be investigated in a subsequent paper.

It could be said that statistical geometry is the inverse to geometric probability, that elegant chapter of the calculus of probability, also related to integral geometry. For presentations of these areas we refer the reader to Blaschke (1955), Deltheil (1920) and Santaló (1936).

2. The circle. We shall begin by the simplest possible case when the image is just a circle, described through the three parameters, the radius $R$ and the coordinates $x_{0}, y_{0}$ of its center. In other words, we have just a three-dimensional image algebra generated from the prototype $=$ unit circle and the group $G$ of similarity transformations mentioned in $\S 1$. 
First, let $x_{0}=y_{0}=0$ and let us estimate $R$. As in the more general case, we could appeal to (1.4). It is interesting that the computational problem has been studied in terms of a numerically efficient algorithm by Bass and Schubert (1967). Since we want algorithms that can be generalized to more challenging image algebras we shall use boundary features instead.

Of course, we could use standard linear-quadratic estimators of these three parameters. However, as we shall see, higher efficiency can be obtained by using boundary features. Also, the methods based on boundary features are more robust and likely to warn us if the shape deviates considerably from a circle.

It seems reasonable that simple algorithms based on diameters of $I^{\mathscr{D}}$ should be informative. By a diameter in the $\phi$-direction is meant the distance between the two support lines of $I^{\mathscr{D}}$ in a direction orthogonal to $\phi$.

It is important to get algorithms that are invariant under $G$, and diameters obviously are. They are also easy to compute. We shall study the properties of such an algorithm below.

Consider the situation described in Figure 2.1. In the unit circle $C$ there are $n$ points distributed as a sample from a uniform probability distribution over $C$. $C$ is centered at the origin and we denote the points by $z_{v}=$ $\left(\xi_{v}, \eta_{v}\right) ; v=1,2, \ldots, n$. Introduce the four stochastic variables

$$
\begin{array}{ll}
\xi_{\min }=\min _{v}\left(\xi_{v}\right), & \eta_{\min }=\min _{v}\left(\eta_{v}\right), \\
\xi_{\max }=\max _{v}\left(\xi_{v}\right), & \eta_{\max }=\max _{v}\left(\eta_{v}\right) .
\end{array}
$$

We want, to begin with, to find their joint distribution function, especially for large values of $n$. It is obvious that, as $n$ increases, $\xi_{\min }, \eta_{\min }$ will tend to -1 and $\xi_{\max }, \eta_{\max }$ to 1 . We therefore normalize them as follows:

$$
\begin{aligned}
& \xi_{\max }=1-\left(u / n^{\alpha}\right), \quad u>0 \\
& \xi_{\min }=-1+\left(v / n^{\alpha}\right), \quad v>0
\end{aligned}
$$

where the particular form of the standardization in terms of the exponent $\alpha$ will be chosen later on. The variables $\eta_{\min }, \eta_{\max }$ will be treated in the same manner.

Consider the function $G$ for $u$ and $v$ :

$$
\begin{aligned}
G(a, b) & =P\{u \geqq a, v \geqq b\} \\
& =P\left\{\xi_{\max } \leqq 1-a / n^{\alpha}, \xi_{\min } \geqq-1+b / n^{\alpha}\right\} \\
& =P\left\{-1+b / n^{\alpha} \leqq \xi_{i} \leqq 1-a / n^{\alpha} ; i=1,2, \ldots, n\right\} .
\end{aligned}
$$

Let the area of the right shaded segment in Figure 2.1 be denoted by $A(d)$, where $x_{2}=1-d$. Then

$$
A(d)=v-(1-d) \sin v
$$




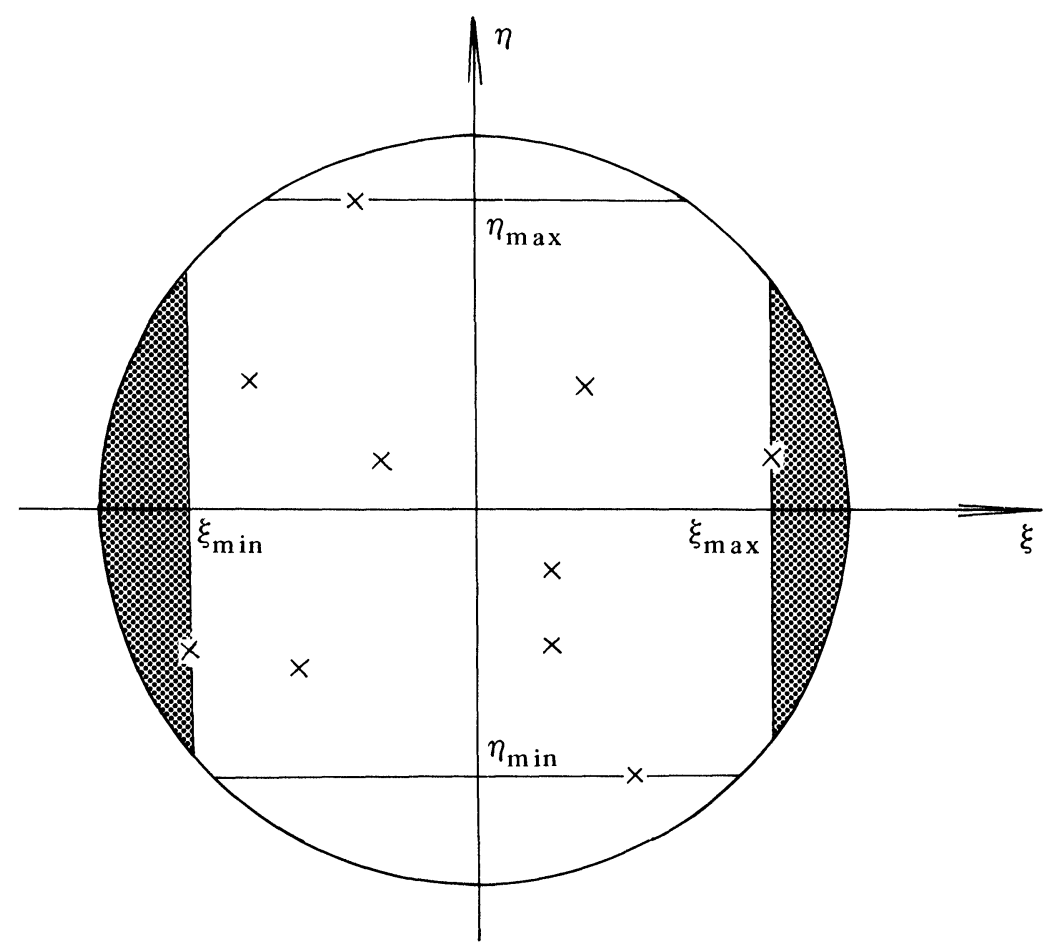

FIGURE 2.1

where $v$ is half the opening angle of the segment so that

$$
v=\arccos (1-d) \text {. }
$$

Then, taking both the shaded segments into account, we get

$$
G(a, b)=\left[\Pi-A\left(a / n^{\alpha}\right)-A\left(b / n^{\alpha}\right)\right]^{n} / \Pi^{n} .
$$

Note, however, that for small values of $d(2.5)$ gives us asymptotically

$$
v \sim \sqrt{2 d}
$$

and

$$
A(d) \sim(4 / 3) \sqrt{2} d^{3 / 2}
$$

Hence the expression inside brackets in (2.6), including the $\Pi^{n}$ factor, can be written asymptotically as

$$
1-\left(4 \sqrt{2} / 2 \Pi n^{3 \alpha / 2}\right)\left(a^{3 / 2}+b^{3 / 2}\right) .
$$


Now it is obvious from the form of (2.9) that $\alpha=2 / 3$ will be the right choice in order to get a nondegenerate limit result. We get then

$$
\lim _{n \rightarrow \infty} G(a, b)=\exp -\frac{4 \sqrt{2}}{3 \Pi}\left(a^{3 / 2}+b^{3 / 2}\right) .
$$

Hence the stochastic variables $u$ and $v$ will be asymptotically independent with the distribution function of Weibull type

$$
H(x)=1-\exp -(4 \sqrt{2} / 3 \Pi) x^{3 / 2}, \quad x>0,
$$

and the frequency function

$$
h(x)=(4 / \Pi \sqrt{2}) \sqrt{x} \exp -(4 \sqrt{2} / 3 \Pi) x^{3 / 2}, \quad x>0 .
$$

Now introduce the empirical first diameter

$$
D_{1}=\xi_{\max }-\xi_{\min },
$$

whose probability distribution is obviously translation-invariant, and the stochastic variable

$$
\lambda_{1}=\left(2-D_{1}\right) n^{2 / 3}=u+v .
$$

It follows that $\lambda$ has asymptotically the frequency function $h * h=k$ with a corresponding distribution function $k=H * H$, so that the resulting distribution is given by

$$
K(x)=\int_{0}^{x} H(x-y) h(y) d y .
$$

The above was done for the first empirical diameter $D_{1}$ in the $x$-direction. We can do the same thing for the second one $D_{2}$ in the $y$-direction and it is not difficult to see that they are asymptotically independent. We can generalize this immediately to $m$ diameters with given, distinct directions $\phi_{1}, \phi_{2}, \ldots, \phi_{m}$. It then follows that, for fixed $m$,

$$
\lim _{n \rightarrow \infty} P\left\{\max _{i} D_{i} \leqq 2-\lambda n^{-2 / 3}\right\}=\lim _{n \rightarrow \infty} P\left\{\min _{i} \lambda_{i} \geqq \lambda\right\}=1-K^{m}(\lambda) .
$$

We now proceed to estimating the unknown radius $R$ of a circle when we have observed the $m$ empirical diameters $D_{1}, D_{2}, \ldots, D_{m}$. The $D_{i}$ have the same asymptotic distribution as before except for the change of scale in the proportion $1: R$ and we can appeal to (2.15) in order to get an estimator $R^{*}$ which is asymptotically median-unbiased.

Hence we have proved the following

THEOREM 2.1. The m-diameter estimator 


$$
R^{*}=\max _{i} D_{i} /\left(2-\lambda n^{-2 / 3}\right)
$$

is asymptotically median-unbiased when $\lambda$ is chosen to satisfy the equation

$$
K(\lambda)=2^{-m}
$$

and with the confidence interval

$$
\max _{i} D_{i} /\left(2-\lambda_{1} n^{-2 / 3}\right) \leqq R \leqq \max _{i} D_{i} /\left(2-\lambda_{2} n^{-2 / 3}\right)
$$

with the confidence level

$$
K^{m}\left(\lambda_{2}\right)-K^{m}\left(\lambda_{1}\right)
$$

A word of caution is needed on the interpretation and application of this theorem. It is tempting to make $m$ large: if we use a many-diameter estimator of $R$ it is likely to be more efficient. This is indeed so, but one should note that in the derivation of the asymptotic result we have used the fact that for large samples it is unlikely that the segments involved overlap. For any value of $m$ overlap can be ruled out with large probability as $n \rightarrow \infty$. If we make $m$ large at the same time the statement, and hence the result in (2.18) and (2.20), will no longer be true. For this reason $m$ should be chosen rather small, say 4 or 6 if $n$ is of the order of magnitude $50-100$.

We now turn to the second part of identifying the image $I$ and try to estimate

$$
g_{\text {trans }}=\left(x_{0}, y_{0}\right)
$$

by estimators $x_{0}^{*}, y_{0}^{*}$ based on $m$ diameters with a set $\Phi$ of $m$ directions $\phi$.

Denote by $\xi_{\max }^{\phi}, \xi_{\min }^{\phi}$ the minimum and maximum respectively measured along the direction $\phi$. With the same notation as before we have

$$
\begin{aligned}
& \xi_{\max }^{\phi}=x_{0} \cos \phi+y_{0} \sin \phi+R-R\left(u / n^{\alpha}\right), \\
& \xi_{\min }^{\phi}=x_{0} \cos \phi+y_{0} \sin \phi-R+R\left(v / m^{\alpha}\right),
\end{aligned}
$$

and we shall use, for the present purpose, their average

$$
\xi^{\phi}=\frac{1}{2}\left[\xi_{\max }^{\phi}+\xi_{\min }^{\phi}\right]=x_{0} \cos \phi+y_{0} \sin \phi+R\left(w / n^{\alpha}\right)
$$

where $w=v-u$. For any set $\Phi$ we know from the last section that the $m$ quantities

$$
(1 / R)\left[\xi^{\phi}-x_{0} \cos \phi-y_{0} \sin \phi\right]
$$

are asymptotically independent, each with the distribution function

$$
G=H * H^{-}
$$

where $H^{-}$denotes the distribution function of $-u$. Note that $G$ is the 
symmetrization of $H$ so that $G-\frac{1}{2}$ is an odd function. The mean of $w$ is of course zero and its variance is $2 \sigma^{2}$.

To identify $x_{0}$ (and similarly $y_{0}$ ) we shall use a linear combination of $\xi^{\phi}$ 's:

$$
x_{0}^{*}=\sum_{\phi \in \Phi} c_{\phi} \xi^{\phi}
$$

where the coefficients $c_{\phi}$ are chosen to make $x_{0}^{*}$ a BLUE. Unbiasedness demands that

$$
\sum c_{\phi} \cos \phi=1, \quad \sum c_{\phi} \sin \phi=0
$$

while the large sample variance is given by

$$
\left(n^{\alpha} / 2 R^{2} \sigma^{2}\right) \operatorname{Var}\left(x_{0}^{*}\right)=\sum c_{\phi}^{2} .
$$

Let us assume a regular arrangement of the $m$ directions,

$$
\Phi=\left\{0, \frac{\Pi}{m}, \frac{\Pi}{m}, \ldots, \frac{(m-1) \Pi}{m}\right\} .
$$

Remembering that $\alpha=2 / 3$, we get

$$
\begin{aligned}
& c_{j}=(2 / m) \cos (j \Pi / m), \quad j=0,1, \ldots, m-1, \\
& \operatorname{Var}\left(x_{0}^{*}\right) \sim 8 R^{2} \sigma^{2} / m n^{4 / 3},
\end{aligned}
$$

which can be used to get confidence intervals for $x_{0}$ and $y_{0}$.

The expression in (2.30) needs some additional remarks. Replacing $I^{\mathscr{D}}$ by the features $R_{1}^{*}, x_{0}^{*}, y_{0}^{*}$ has been done using boundary features for $I^{\mathscr{D}}$ : quantities derived from $\xi_{\max }^{\phi}, \xi_{\min }^{\phi}$ and combining them linearly. We have used quasi-linear feature extraction.

If instead we had used the linear features $\bar{x}, \bar{y}$, i.e. the coordinates of the centroid of $I^{\mathscr{D}}$, we would have gotten a variance of the order of magnitude $n^{-1}$. We have gained in recognition accuracy by a factor of the order $n^{1 / 3}$ by preferring the quasi-linear boundary features.

The case when $\mathscr{T}$ consists only of circles is of course quite simple. Let us therefore turn to the situation when we generate the images by $I=g V$, where the prototype $V$ is a closed, convex figure with continuous curvature. If we had allowed corners the following analysis would have to be modified, but we shall postpone a discussion of how this could be done here. Let us start by only having $G=$ scale changes, so that we have to identify a scale factor, say $R$. Let us put the area of $V$ equal to $A$.

Consider Figure 2.2 where $T_{1}$ and $T_{2}$ are the two support lines in a direction $\phi$. Let us denote by $R_{1}(\phi)$ and $R_{2}(\phi)$ the radii of curvature at the points of contact $P_{1}$ and $P_{2}$. Introduce a coordinate axis $\xi$ perpendicular to $\phi$. 


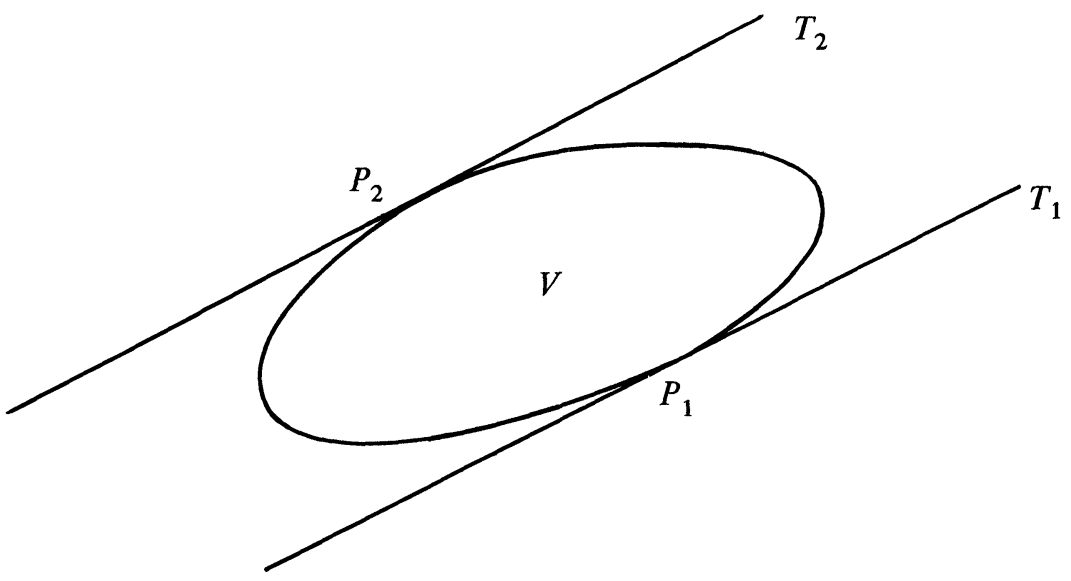

FigURE 2.2

For the deformed image $I^{\mathscr{D}}$ we introduce $\xi_{\min }^{\phi}, \xi_{\max }^{\phi}$ analogous to the quantities used in the case of the circle (see Figure 2.1). We then get

$$
\begin{aligned}
& \xi_{\min }^{\phi}=R\left(x_{1}^{\phi}+\sigma_{1}(\phi) u / n^{\alpha}\right) \\
& \xi_{\max }^{\phi}=R\left(x_{2}^{\phi}-\sigma_{2}(\phi) v / n^{\alpha}\right)
\end{aligned}
$$

where the factors $\sigma_{i}(\phi)$ measure the size of a small segment at the points of contact $P_{i}$ so that

$$
\sigma_{i}(\phi)=\frac{\Pi R_{1}^{2}(\phi)}{\text { area of } V}=\frac{\Pi R_{i}^{2}(\phi)}{A} .
$$

The quantity

$$
R_{\phi}^{*}=\left(\xi_{\max }^{\phi}-\xi_{\min }^{\phi}\right) \div\left(x_{2}^{\phi}-x_{1}^{\phi}\right)
$$

can then be written as

$$
R_{\phi}^{*}=R+\frac{\left[\sigma_{1}(\phi) u+\sigma_{2}(\phi) v\right] R}{n^{\alpha}\left(x_{2}^{\phi}-x_{1}^{\phi}\right)} .
$$

Combining $m$ such values into a new feature

$$
R^{*}=\sum c_{\phi} R_{\phi}^{*},
$$

we choose the coefficients $c_{\phi}$ so that

$$
\sum c_{\phi}\left(1+\mu \frac{\sigma_{1}(\phi)+\sigma_{2}(\phi)}{n^{\alpha} x_{2}^{\phi}-x_{1}^{\phi}}\right)=\sum c_{v} \alpha_{v}=1
$$

where $\mu$ is the expected value of the $H$-distribution, and 


$$
\sum c_{\phi}^{2} \frac{\sigma_{1}(\phi)+\sigma_{2}(\phi)}{\left(x_{2}^{\phi}-x_{1}^{\phi}\right)^{2}}=\sum c_{\phi}^{2} \beta_{v}=\min _{(2.39)}
$$

defining $\alpha_{v}$ and $\beta_{v}$ in the obvious manner. This gives us immediately

$$
c_{\phi}=\frac{\alpha_{v} \beta_{v}}{\sum \alpha_{v}^{2} / \beta_{v}}=c\left(\alpha_{v} / \beta_{v}\right), \quad c=\frac{1}{\sum \alpha_{v}^{2} / \beta_{v}}
$$

with the resulting variance

$$
\operatorname{Var}\left(R^{*}\right) \sim \frac{R^{2} \sigma^{2}}{n^{2 \alpha}} \frac{1}{\sum \alpha_{v}^{2} / \beta_{v}} .
$$

A problem that does not arise for the circle but for the present prototype $V$ is when $G=$ rotation group. Say that the scale and location of $I$ have already been determined and use the notation introduced in connection with Figure 2.2. We shall use the notation $\theta$ for the unknown angle of rotation. Then the set of $2 m$ quantities

$$
\delta_{1}^{\phi}=\frac{x_{1}(\phi+\theta)-\xi_{\min }^{\phi}}{\sigma_{1}(\phi+\theta)} n^{\alpha}, \quad \delta_{2}^{\phi}=\frac{\xi_{\max }^{\phi}-x_{2}(\phi+\theta)}{\sigma_{2}(\phi+\theta)} n^{\alpha}
$$

are all asymptotically independent and distributed according to the $H$-distribution with mean $\mu$. It is therefore natural to estimate $\theta$ by solving the least-squares problem

$$
\min _{\theta} \sum_{\phi \in \Phi}\left(\delta_{1}^{\phi}-\mu\right)^{2}
$$

This is a nonlinear least-squares problem but for the asymptotic solution it is enough to linearize the functions of $\theta$ appearing in the $\delta_{i}^{\phi}$. We have assumed here that the $2 m$ values of $x_{i}(\phi+\theta)$ determine $\theta$ uniquely.

We can now allow the image algebra $\mathscr{T}$ to be more extensive. Let the prototype again be the unit disk and $G=$ \{translations, scale changes . If $\mathscr{T}$ has as a binary operation unions of sets $U$ we can get images like the one in Figure 2.3a. If only two prototypes are allowed in any image we have a six-dimensional image algebra: four coordinates for the centers and two for the radii. An ad hoc identification procedure based on the centroid and moments of inertia would not suffice.

We can, however, base the identification on boundary statistics by extending the approach that we have used. The methodology is applicable if we remember to take account of the special case that arises when we approach $I$ from the direction of one of the two common tangents to both circles. If we do we get two contributions to the distribution of $\xi_{\max }^{\phi}, \xi_{\min }^{\phi}$ rather than one: we have two points where the tangent touches $I$.

If instead we allow as binary operation intersection of sets and as 

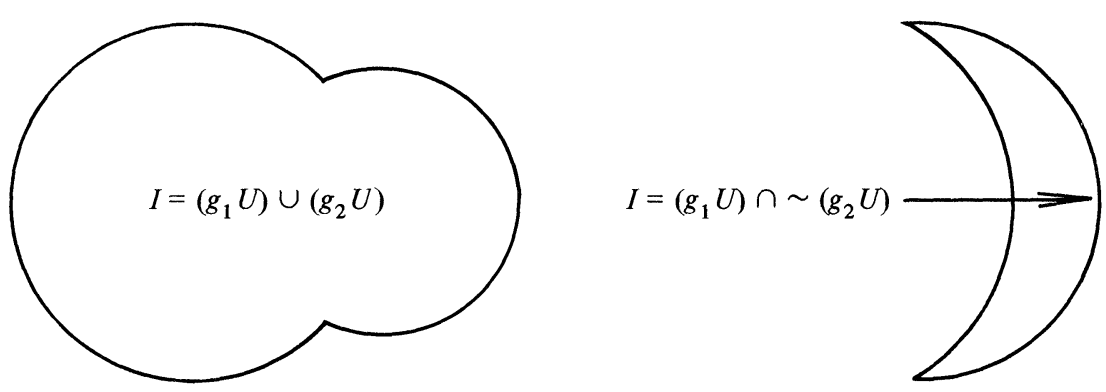

FIGURE 2.3

unary operation complementation we can get crescent-shaped images, as the one in Figure 2.3b. Here our methodology must be extended to be able to handle images with corners.

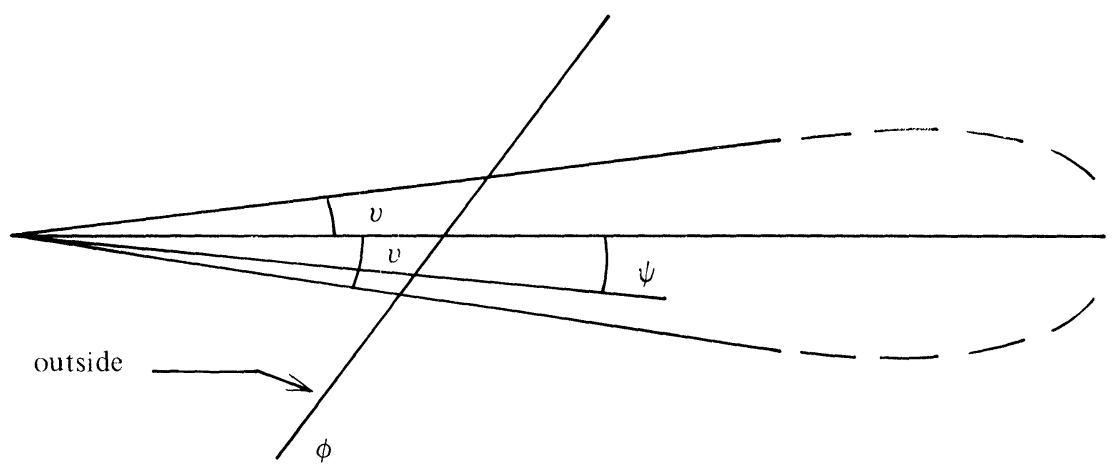

FIGURE 2.4

To do this consider the corner in Figure 2.4 with an opening angle $2 v$ and we approach $I^{\mathscr{D}} \subset I$ from the direction $\phi$, in this case more conveniently expressed through the angle $\psi$ in the figure. Denoting the total area of $I$ by $A=m(I)$, we get for the probability that a point drawn at random from $I$ will fall to the left of $\phi$ (see Figure 2.4), which is asymptotically correct for small $x$ assuming continuous curvature away from the corner,

$$
\left(x^{2} / 2 A\right)[\sin (v+\phi)+\sin (v-\phi)]=\left(x^{2} / A^{2}\right) \sin v \cos \phi .
$$

It is now clear how to normalize $x$. Let us put

$$
x=u / \sqrt{n},
$$


which leads to the asymptotic distribution function for $u$

(2.42) $\lim _{n \rightarrow \infty} P(n \leqq t)=1-\exp \left(-\frac{t^{2}}{A^{2}} \cos \phi \cos v\right), \quad t>0$

This half-Gaussian distribution can be used for the treatment of images with corners instead of the $H$-distribution, which is valid for smooth boundaries.

As we go ahead to more extensive image algebras formed in the same manner with higher $\operatorname{dim}(\mathscr{T})$ we can, in principle, apply the same method. For high dimensionality they do not seem as natural, however, and one would prefer more nonparametric methods. As a matter of fact, already the six-dimensional crescents may present such a case. We shall describe some alternative identification procedures.

Start from $\mathscr{T}=$ crescent $\}$; let us form the convex hull of $I^{\mathscr{D}}$ (see Figure $2.5)$, and denote the lengths of the sides by $l_{i}$ and their orientations by $\alpha_{i}$.

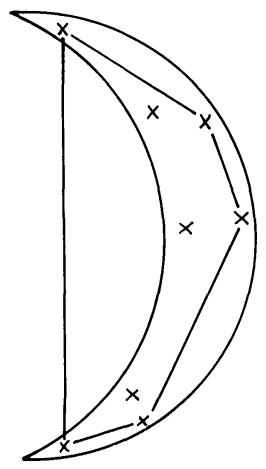

Figure 2.5

Consider the end points $P^{\prime}$ and $P^{\prime \prime}$ of the side with greatest $l_{i}$ and introduce the circles $C\left(P^{\prime}, P^{\prime \prime} ; \rho\right)$ of radius $\rho$ passing through $P^{\prime}$ and $P^{\prime \prime}$.

Form an image of the form

$$
I^{*}=C\left(P^{\prime}, P^{\prime \prime} ; \rho_{1}\right) \cap \sim C\left(P^{\prime}, P^{\prime \prime}, \rho_{2}\right)
$$

and choose $\rho_{1}$ and $\rho_{2}$ so that $m\left(I^{*}\right)=$ min with the constraint $I^{*} \subset I$. One can prove that this is a consistent estimator of $I$.

Another boundary statistic that could be exploited is the set of quantities

$$
\rho_{i}^{*}=2\left(\alpha_{i+1}-\alpha_{i}\right) /\left(l_{i+1}+l_{i}\right) .
$$

They estimate the curvature and could be used both to estimate the inverses $1 / R_{1}, 1 / R_{2}$ and to find the location of the centers of the two circles. 
3. Breakpoints in the boundary. The function of boundary features is to catch some typical property of the true boundary $\partial I$ mirrored through $\mathscr{D}$ into the "boundary" of the deformed image $I^{\mathscr{D}}$. Such properties can be the value of the radius of curvature, more or less sudden changes in the curvature, or corners. We shall study the recognition of one such property in this section.

Consider an image

$$
I=\text { Half-strip } \cup \text { Wedge }
$$

generated from a half-strip and an infinite wedge, resulting in the shape in Figure 3.1, where the unknown parameter $u$ expresses the location of the breakpoint in $\partial I$ where the wedge meets the half-strip.

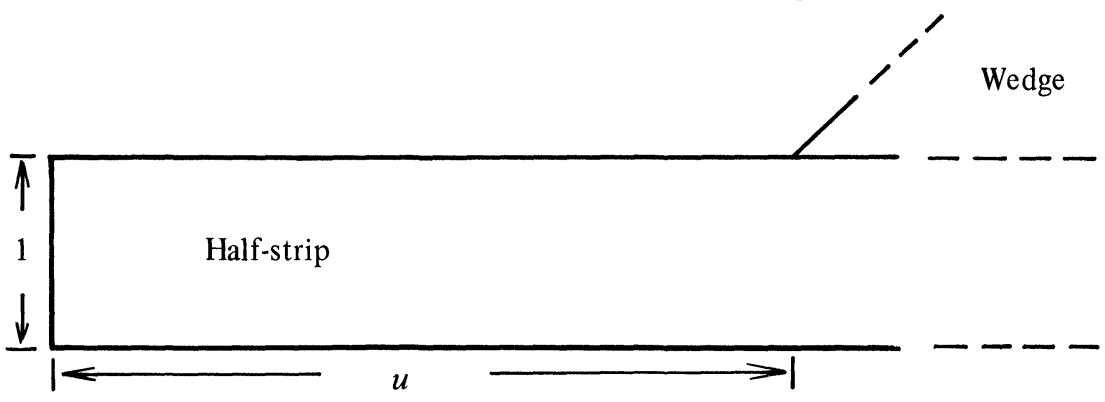

Figure 3.1

We shall investigate two deformation mechanisms, one version of $\mathscr{D} 1$ and also $\mathscr{D} 2$.

First, let the infinite sequence of independent stochastic variables $x_{1}$, $x_{2}, x_{3}, \ldots$ be distributed as $R\left(0, a_{i}\right)$. We shall assume that

$$
a_{i}=1+(1 / n) \phi(i / n)
$$

where $\phi$ is a given nondecreasing and piecewise continuous function. In the special case belonging to (3.1) the $a_{i}$ are constant and equal to one up to some large subscript $i_{0}=[\alpha n]$, and we want to find out where the breakpoint $i_{0}$ is, having observed the $x_{1}$. Our procedure will be based on the first observation $x_{k}$ that passes through a level $l>1$ with

$$
l=1+(\lambda / n), \quad \lambda>0
$$

so that

$$
x_{1} \leqq l, \quad x_{2} \leqq l, \ldots, \quad x_{k-1} \leqq l, \quad x_{k}>l .
$$

The probability $p_{k}$ of $(3.4)$ is

$$
p_{k}=\prod_{1}^{k-1} P\left(x_{i} \leqq l\right) \cdot P\left(x_{k}>l\right)
$$




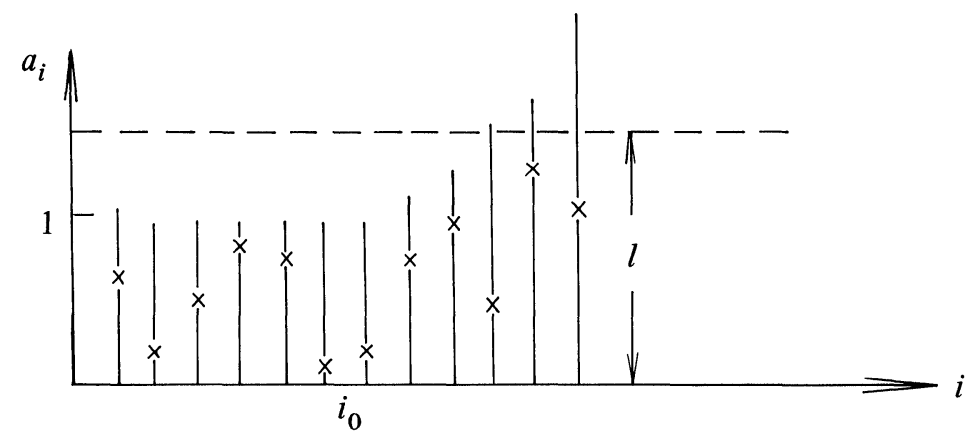

FIGURE 3.2

and the distribution for the normalized subscript $x=k / n$ is

$$
F_{n}(x)=P(k \leqq n x)=p_{1}+p_{2}+\cdots+p_{[n x]}=1-Q_{[n x]}
$$

where

$$
Q_{k}=\prod_{1}^{k} P\left(x_{i} \leqq l\right)
$$

Using the rectangular distribution we notice that the factors in (3.7) are 1 for $i$ 's satisfying

$$
\phi(i / n) \leqq \lambda .
$$

If we denote by $x_{0}$ the smallest root of $\phi(x)=\lambda$ we get

$$
Q_{[n x]}=\prod_{x_{0} \leqq(i / n) \leqq x} P\left(x_{i} \leqq l\right)=\prod_{x_{0} \leqq(i / n) \leqq x} \frac{1+\lambda / n}{1+(1 / n) \phi(i / n)} .
$$

Hence for large values of $n$

$$
\log Q_{[n x]}=\frac{1}{n} \sum_{x_{0} \leqq(i / n) \leqq x}\left[\lambda-\phi\left(\frac{i}{n}\right)\right]+o\left(\frac{1}{n}\right)
$$

and we get

THEOREM 3.1. With (3.2), (3.3), the limiting distribution $F$ of the time $k$ when the observations pass through the level lis given by

(3.11) $F(x)=\lim _{n \rightarrow \infty} P\{k \leqq n x\}=1-\exp \left(\int_{x_{0}}^{x}[\lambda-\phi(x)] d x\right), \quad x \geqq x_{0}$.

To apply this to the case in Figure 3.2 we just put

$$
\begin{aligned}
\phi(x) & =0, & & 0 \leqq x \leqq \alpha, \\
& =\beta(x-\alpha), & & \alpha \leqq x,
\end{aligned}
$$


where $\beta$ is the slope of the linear trend in the figure. We then get

$$
x_{0}=\alpha_{+} \lambda / \beta
$$

and we have

Corollary 3.1. For $a_{i}$ as given in Figure 3.2 we get the limit distribution

$$
F(x)=1-\exp \left(-\frac{\beta}{2}(x-\alpha)^{2}+\left(x-\alpha-\frac{\lambda}{\beta}\right)+\frac{\lambda^{2}}{2 \beta}\right), x>x_{0} .
$$

To get the $q$-quantile for the error $y=x-\alpha$ we have to put

$$
q=F\left(y_{q}+\alpha\right)
$$

so that

$$
y_{q}=(\lambda / \beta)+\sqrt{-(2 / \beta) \log (1-q)}
$$

so that the median of $x$ is

$$
\operatorname{Median}(x)=\alpha+y_{5}=\alpha+(\lambda / \beta)+\sqrt{(2 / \beta) \log 2}
$$

and we should use the corrected diameter

$$
x-\lambda / \beta-\sqrt{(2 / \beta) \log 2} .
$$

Let us instead look for the maximum likelihood estimator assuming $\phi(x)=1$ for $0 \leqq x \leqq \alpha$ and $\phi(x)=1+d$ for $x \geqq \alpha$ with $d>\lambda$. Then we get from Theorem 3.1 the following limit result.

COROLlary 3.2. For a sudden change of the $a_{i}$ as described above the limiting distribution is given by

$$
F(x)=1-\exp [-(d-\lambda)(x-\alpha)], \quad x>\alpha .
$$

Let us instead look for the maximum likelihood estimator assuming that we have access to $N=[c n], c>\alpha$, observations. We still let $\alpha$ represent the true (but unknown) value of the parameter in (3.12) and consider the likelihood function in $\alpha^{\prime}, \alpha^{\prime}>\alpha$,

$$
L\left(\alpha^{\prime}\right)=\prod_{i=\left[\alpha^{\prime} n\right]}^{[c n]} \frac{1}{1+(\beta / n)\left(i / n-\alpha^{\prime}\right)}
$$

if $x_{i} \leqq 1+(\beta / n)\left(i / n-\alpha^{\prime}\right)$ for $\left[\alpha^{\prime} n\right] \leqq i \leqq[c n]$, otherwise $L\left(\alpha^{\prime}\right)=0$. Hence the maximum likelihood estimation is the largest value of $\alpha^{\prime}$ such that

$$
x_{i} \leqq 1+(\beta / n)\left(i / n-\alpha^{\prime}\right), \quad\left[\alpha^{\prime} n\right] \leqq i \leqq[c n] .
$$

Therefore the event $\alpha^{*} \geqq \alpha^{\prime}$ is the same as the event in 1 , so that the 
distribution function of $\alpha^{*}$ is

$$
\begin{aligned}
G_{n}(t) & =P\left\{\alpha^{*} \leqq t\right\} \\
& =1-\prod_{\alpha \leqq(i / n) \leqq t} \frac{1}{1+(\beta / n)(i / n-\alpha)} \prod_{t<(i / n) \leqq c} \frac{1+(\beta / n)(i / n-t)}{1+(\beta / n)(i / n-\alpha)}
\end{aligned}
$$

so that

$$
\begin{aligned}
G(t)=\lim _{n \rightarrow \infty} G_{n}(t) & =1-\exp -\beta\left\{\int_{\alpha}^{t}(x-\alpha) d x+\int_{t}^{c}(t-\alpha) d x\right\} \\
& =1-\exp -\beta\left[\frac{(t-\alpha)^{2}}{2}+(c-t)(t-\alpha)\right] .
\end{aligned}
$$

Hence we have the following result.

THEOREM 3.2. The maximum likelihood estimator has the limiting distribution

$$
\begin{aligned}
G(t) & =1-\exp -\beta\left[(t-\alpha)^{2} / 2+(c-t)(t-\alpha)\right], \quad \alpha \leqq t \leqq c, \\
& =1, \quad t>c .
\end{aligned}
$$

We can now extend the results in several directions. First, assume that $x_{i}$ is not $R\left(0, a_{i}\right)$ but is the largest of a sample of $s$ observations from $R\left(0, a_{i}\right)$. The only thing that changes in our derivations is that the ratios in the second product of (3.9) should be raised to the sth power. Instead of (3.11) we get the limiting distribution

$$
1-\exp \left(s \int_{x_{0}}^{x}[\lambda-\phi(x)] d x\right), \quad x>x_{0}
$$

and similar changes in the other results above.

Now let us study the situation depicted in Figure 3.3, where the image is defined by the boundaries $x=0$ and $x=1$ and

$$
\phi_{1}(x)-\alpha_{1} \leqq y \leqq \phi_{2}(x)+\alpha_{2}, \quad 0 \leqq x<1,
$$

where the parameters $\alpha_{1}$ and $\alpha_{2}$ are nonnegative and $\phi_{1}(x) \leqq \phi_{2}(x)$ for all $x \in(0,1) . I^{\mathscr{D}}$ is generated as before by a Poisson process with intensity $\mu$, resulting in the $n$ points with coordinates $\left(x_{1}, y_{1}\right),\left(x_{2}, y_{2}\right), \ldots,\left(x_{n}, y_{n}\right)$.

This situation corresponds to a pure image generated as

$$
I=\left(g_{1} s_{1}\right) \cap\left(g_{2} s_{2}\right)
$$

where the signs $s_{1}, s_{2}$ are defined by

$$
\begin{aligned}
& s_{1}=\left\{(x, y) \mid 0 \leqq x \leqq 1, y \leqq \phi_{2}(x)\right\} \\
& s_{2}=\left\{(x, y) \mid 0 \leqq x \leqq 1, y \geqq \phi_{1}(x)\right\}
\end{aligned}
$$




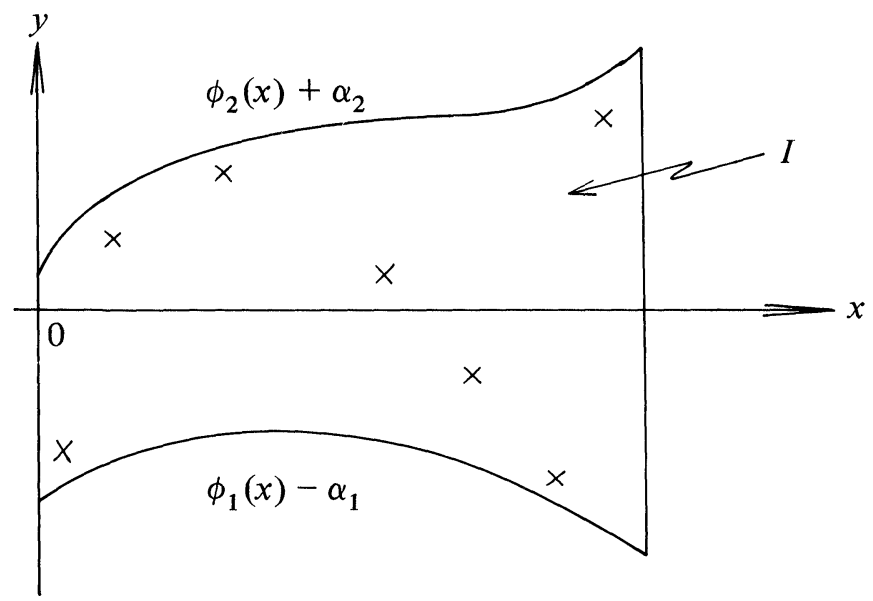

FIGURE 3.3

and the $g$ 's are translations in the $y$-direction.

As before, the ML estimates $\alpha_{1}^{*}, \alpha_{2}^{*}$ are given by minimizing the area $A\left(\alpha_{1}, \alpha_{2}\right)$ of $I$ with the constraint that all $\left(x_{v}, y_{v}\right) \in I$. We have

$$
A\left(\alpha_{1}, \alpha_{2}\right)=A(0,0)+\alpha_{1}+\alpha_{2}
$$

so that to minimize $A$ means minimizing $\alpha_{1}+\alpha_{2}$ and can be done by minimizing $\alpha_{1}$ and $\alpha_{2}$ separately because of the form of the constraint.

$$
\alpha_{1}^{*}=\max _{i}\left[\phi_{1}\left(x_{i}\right)-y_{i}\right], \quad \alpha_{2}^{*}=\max _{i}\left[y_{i}-\phi_{2}\left(x_{i}\right)\right] .
$$

To find the joint distribution function $F$ for $\left(\alpha_{1}^{*}, \alpha_{2}^{*}\right)$ we note that, for $s \leqq \alpha_{1}, t \leqq \alpha_{2}$,

$$
\begin{aligned}
F(s, t) & =P\left(\alpha_{1}^{*} \leqq s, a_{2}^{*} \leqq t\right) \\
& =P\left(\phi_{1}\left(x_{i}\right)-y_{i} \leqq s \text { and } y_{i}-\phi_{2}\left(x_{i}\right) \leqq t \text { for all } i\right) \\
& =P\left(\phi_{1}\left(x_{i}\right)-s \text { and } y_{i} \leqq \phi_{2}\left(x_{i}\right)+t \text { for all } i\right) \\
& =\exp \left(-\mu\left[A\left(\alpha_{1}, \alpha_{2}\right)-A(s, t)\right]\right)
\end{aligned}
$$

so that

$$
F(s, t)=\exp \left(-\mu\left(\alpha_{1}-s\right)-\mu\left(\alpha_{2}-s\right)\right) .
$$

THEOREM 3.3. The maximum likelihood estimators $\alpha_{1}^{*}$, $\alpha_{2}^{*}$ for the image of form (3.34) viewed after Poisson deformation mechanism has been applied are given by (3.36) and are stochastically independent and $\alpha_{i}-\alpha_{i}^{*}$ is exponentially distributed with mean $1 / \mu, i=1,2$. 
4. Behavior of the leeway set. With the same notation as before, let $I$ be an image of the form $g I_{0}$ for a given and fixed prototype $I_{0}$ which we shall take as a convex set with a boundary which has continuous and positive curvature. Let $G$ be the whole or a subgroup of the Euclidean group on $R^{2}$. The deformation mechanism $\mathscr{D}$ results in a sample $\left(x_{1}, y_{1}\right)$, $\left(x_{2}, y_{2}\right), \ldots,\left(x_{n}, y_{n}\right)$ of points from $I$. We shall also assume that the group element $g$ associated with the pure image is uniquely determined so that $g_{1} I_{0}=g_{2} I_{0}$ implies $g_{1}=g_{2}$.

The only possible values of $g$ are the ones satisfying

$$
I^{\mathscr{D}} \subset I=g I_{0}
$$

or

$$
g^{-1}\left(x_{v}, y_{v}\right) \in I_{0} \quad \text { for } v=1,2, \ldots, n .
$$

Let us denote the subset of $G$ given by (4.1) by $\gamma_{n}=\gamma\left(I^{\mathscr{D}}\right)$. Any reasonable algorithm for recognizing $g$ must give a result in $\gamma$ and deciding between all such admissible algorithms will be based on some additional criterion such as maximum likelihood or mean square error with some distance on $G$.

The size of $\gamma_{n}$ determines how much leeway we have in picking a value of $g$ and this is what we shall call the set $\gamma_{n}$. Notice that $\gamma_{n}$ are stochastic sets which form a nonincreasing sequence in $n$. Hence as $n$ tends to infinity the $\gamma_{n}$ tend to a limiting set $\lim _{n \rightarrow \infty} \gamma_{n}$.

LEMMA 4.1. We have, with probability one, $\lim _{n \rightarrow \infty} \gamma_{n}=g_{\text {true }}$ so that in the limit the leeway contains only the true value $g_{\text {true }}$.

Proof. Let $\overline{\bar{I}}^{\mathscr{D}}$ stand for the convex hull of $\gamma_{n}$. Then $\overline{\bar{I}}^{\mathscr{D}} \rightarrow I=$ $g_{\text {true }} I_{0}$, with probability one. Then (4.2) is equivalent to the set of $g$ 's such that

$$
\left\{g \mid g^{-1} \overline{\bar{I}}^{\mathscr{D}} \subset I_{0}\right\}=\gamma_{n}
$$

Hence

$$
\lim _{n \rightarrow \infty} \gamma_{n}=\left\{g \mid g^{-1} g_{\text {true }} I_{0}=I_{0}\right\}
$$

which implies $g^{-1} g_{\text {true }}=e=$ unit element of $G$ and $g=g_{\text {true }}$ is the only element of the limiting set.

REMARK. Lemma 4.1 means that, without appealing to any additional optimality criterion, any admissible identification algorithm is consistent in the statistical sense.

Let us look at two special cases. First let $G$ be just the group of translations of the plane. Then, writing $g=(a, b), g_{\text {true }}=(\alpha, \beta)$, 


$$
\gamma_{n}=\left\{(a, b) \mid\left(x_{v}-a, y_{v}-b\right) \in I_{0} ; v=1,2, \ldots, n\right\} .
$$

This means we can write the leeway set as

$$
\gamma_{n}=\bigcap_{v=1}^{n} S_{v}
$$

where the $S_{v}$ 's are stochastically independent random sets and can be written, with obvious notation,

$$
S_{v}=\left\{(a, b) \mid(a, b) \in\left(x_{v}, y_{v}\right)-I_{0}\right\} .
$$

It is clear that $\gamma_{n}$ is also convex.

On the other hand, if $G$ is the linear group (for simplicity we shall only deal with the proper linear group where the determinant is positive), so that $g^{-1}$ can be represented as a $2 \times 2$ nonsingular matrix,

$$
g^{-1}=\left\{\begin{array}{ll}
g_{11} & g_{12} \\
g_{21} & g_{22}
\end{array}\right\}
$$

then the leeway set is still of the form (4.6) but now with

$$
S_{v}=\left\{g \mid\left(g_{11} x_{v}+g_{12} y_{v}, g_{21} x_{v}+g_{22} y_{v}\right) \in I_{0}\right\}
$$

(it is convenient to work with $g^{-1}$ rather than with $g$ ). If we represent our convex set $I_{0}$ in terms of its supporting half-planes

$$
I_{0}=\bigcap_{\rho \in \Phi}\left\{(x, y) \mid a_{\phi} \leqq l_{1}^{\phi} x+l_{2}^{\phi} y \leqq b_{\phi}\right\}
$$

then the leeway set can be expressed as

$$
\begin{array}{r}
\gamma_{n}=\left\{g \mid a_{\phi} \leqq l_{1}^{\phi}\left(g_{11} x_{v}+g_{12} y_{v}\right)+l_{2}^{\phi}\left(g_{21} x_{v}+g_{22} y_{v}\right) \leqq b_{\phi} ;\right. \\
\text { all } \phi \in \Phi ; \text { all } v=1,2, \ldots, n\} .
\end{array}
$$

Hence $\gamma_{n}$ is again convex.

Lemma 4.2. In the case (4.8) ML estimation of $g$ amounts to solving a quadratic programming problem.

Proof. The likelihood function is, for all $\left(x_{v}, y_{v}\right)$ in the pure image (otherwise it vanishes),

$$
L=A^{-n}
$$

where $A$ is the area of $I$ :

$$
A=m(I)=\operatorname{det}(g) \cdot m\left(I_{0}\right)=\operatorname{det}(g) \cdot A_{0} .
$$

In other words, we have to minimize the quadratic function 


$$
\operatorname{det}(g)=g_{11} g_{22}-g_{12} g_{21} .
$$

We shall now study in more detail how $\gamma_{n}$ tends toward the empty set and we shall do it under the assumptions given above and for the additive group of translations.

THEOREM 4.1. The expected area of the leeway set $\gamma_{n}$ satisfies

$$
\lim _{n \rightarrow \infty} n^{2} E\left[m\left(\gamma_{n}\right)\right]=2 A_{0}^{2} \int_{0}^{\pi} \frac{d \phi}{D_{\phi}^{2}}
$$

where $A_{0}$ is the area of $I_{0}$ and $D_{\phi}$ the diameter associated with support lines in the direction $\phi$.

Proof. Let $f$ be the indicator function of $I_{0}$. Then the indicator function of $\gamma_{n}$ can be written, using (4.6) and (4.7),

$$
\phi(x, y)=\prod_{v=1}^{n} f\left[x_{v}-x, y_{v}-y\right]
$$

so that

$$
m\left(\gamma_{n}\right)=\iint \phi(x, y) d x d y=\iint \prod_{v=1}^{n} f\left[\left(x_{v}-x, y_{v}-y\right)\right] d x d y
$$

But the points $\left(x_{v}, y_{v}\right)$ are stochastically independent with the frequency function $1 / A_{0} \cdot f(x, y)$ so that

$$
\begin{aligned}
\operatorname{Em}\left(\gamma_{n}\right) & =\iint\left\{\frac{1}{A_{0}} \iint f[u-x, v-y] f(u, v)\right\}^{n} d x d y \\
& =\iint K^{n}(x, y) d x d y .
\end{aligned}
$$

It is clear, since $K(x, y)$ is $1 / A_{0}$ times the area of the intersection of $I_{0}$ with $I_{0}+(x, y)$, that this kernel is bounded by one, which value is attained for $x=y=0$.

If the vector $(x, y)$ is small and points in the direction $\phi$ we have

$$
K(x, y)=1-\sqrt{x^{2}+y^{2}} / A_{0} D_{\phi}(1+o(1))
$$

where $D_{\phi}$ is the diameter associated with support lines in the direction $\phi$. Hence

$$
\lim _{n \rightarrow \infty} n^{2} \operatorname{Em}\left(\gamma_{n}\right)=\iint \exp \left[-\frac{1}{A_{0}} D_{\phi} \sqrt{x^{2}+y^{2}}\right] d x d y
$$

or, in polar coordinates, 


$$
\begin{aligned}
\lim _{n \rightarrow \infty} n^{2} \operatorname{Em}\left(\gamma_{n}\right) & =\int_{\phi=0}^{2 \pi} \int_{\rho=0}^{\infty} \exp \left[-\frac{1}{A_{0}} D_{\phi} \rho\right] \rho d \rho d \phi \\
& =A_{0}^{2} \int_{0}^{2 \pi} \frac{d \phi}{D_{\phi}^{2}}
\end{aligned}
$$

This tells us at what rate we can expect the area of the leeway set to decrease. We can also find out how the linear dimensions decrease, and we state the result without proof.

THEOREM 4.2. The distribution function $F_{n}$ of the linear dimension $l$ in the direction $\phi$

$$
l=\max \left\{s \mid(\alpha+s \cos \phi, \beta+s \sin \phi) \in \gamma_{n}\right\}
$$

satisfies the limit relation

$$
\lim _{n \rightarrow \infty} F_{n}(n t)=1-\exp -\frac{t D_{\phi}}{A_{0}} .
$$

REMARK. The theorem says that the standardized linear extent in the $\phi$-direction, $n l_{\phi}$, has asymptotically an exponential distribution with mean $=A_{0} / D_{\phi}$. But this implies that we cannot expect statistical stability for $n l_{\phi}$ even though $n$ is quite large. It would be of interest to see if the correlation coefficient for $l_{\phi}$ and $l_{\psi}$ converges and if so, if the limit is different from zero, for $\phi \neq \psi$, but this has not yet been done.

Now let $G$ be the set of rotations around some point inside $I_{0}$ whose boundary, represented in polar coordinates, is

$$
\rho=\rho(\phi), \quad 0 \leqq \phi<2 \pi,
$$

so that the leeway set $\gamma_{n}$ is expressed in terms of the angle $\phi$ of rotation by

$$
\gamma_{n}=\left\{\phi \mid R^{\phi}\left(x_{v}, y_{v}\right) \in I_{0} ; v=1,2, \ldots, n\right\}
$$

and $R^{\phi}$ stands for the rotation operator. Let $f$ be the indicator function of $I_{0}$ :

$$
K_{n}(\phi)=\left(1 / A_{0}\right) m\left(I_{0} \cap R^{-\phi} I_{0}\right) ;
$$

we have, however,

$$
m\left(I_{0} \cap R^{-\phi} I_{0}\right)=\frac{1}{2} \int \min ^{2}[\rho(\psi), \rho(\psi+\phi)] d \psi .
$$

We shall assume

$$
\begin{aligned}
& \rho \in C_{2}, \\
& \rho^{\prime}(\psi) \leqq 0, \quad \text { in intervals }\left(a_{k}, b_{k}\right), \\
& \rho^{\prime}(\psi)>0, \quad \text { for other } \phi^{\prime} \mathrm{s},
\end{aligned}
$$


so that the contribution to the integral in (4.30) from $\left(a_{k}, b_{k}\right)$ is, for small $\phi>0$,

$$
\frac{1}{2} \int_{a_{k}}^{b_{k}}\left[\rho^{2}(\psi)+2 \phi \rho(\psi) \rho^{\prime}(\psi)\right] d \psi+o(\phi) .
$$

The other intervals give just the contribution

$$
\frac{1}{2} \int \rho^{2}(\psi) d \psi+o\left(\phi^{2}\right)
$$

Hence, for $\phi>0$,

$$
m\left(I_{0} \cap R^{-\phi} I_{0}\right)=A_{0}+\frac{\phi}{2} \sum_{k}\left[\rho^{2}\left(b_{k}\right)-\rho^{2}\left(a_{k}\right)\right]
$$

and, for $\phi<0$, a similar expression but with the sum evaluated over the remaining intervals. Let us denote these two sums by $\sigma^{-}$and $\sigma^{+}$respectively. Combining this with (4.29), (4.30) and (4.31) we get the same method as for Theorem 4.1.

THEOREM 4.3. For the leeway set $\gamma_{n}$ expressed in the angle of rotation we get, when $G$ is the rotation group,

$$
\lim _{n \rightarrow \infty} n E\left[m\left(\gamma_{n}\right)\right]=2 A_{0}\left(\frac{1}{\sigma^{+}}-\frac{1}{\sigma^{-}}\right) .
$$

It may be possible to treat the case when $G=$ scale changes (not necessarily the same in the $x$ and $y$ directions) by the same method.

5. General recognition algorithms. So far we have employed algorithms based on boundary features for recognition. Is it possible to construct more general algorithms that do not depend upon the algebraic structure of the image algebra $\mathscr{T}$ ? To judge the performance of such an algorithm we shall require, as a minimum, that it is consistent in the sense that the expected error tends to zero with $n, \lambda, \lambda_{1}$ or $\lambda_{2}$ respectively for the $\mathscr{D}$ 's described in $\$ 1$. By error we mean the Lebesgue measure of the symmetric difference between the pure and the recognized images:

$$
\text { error }=e\left(I, I^{*}\right)=m\left(I \Delta I^{*}\right)=m\left[\left(I \cap \sim I^{*}\right) \cup\left(I^{*} \cap \sim I\right)\right] .
$$

It is obvious that $I^{\mathscr{D}}$ itself cannot be used as $I^{*}$ since $e\left(I, I^{\mathscr{D}}\right)=m(I)$ and does not tend to zero. The reason is, of course, that $I^{\mathscr{D}}$ is too sparse; we need more "solid" sets as recognizers of $I$.

Consider instead

$$
I^{*}=\bigcup_{v=1}^{n} C_{\rho}\left(z_{v}\right)
$$


where $C_{\rho}(z)$ stands for the disk with radius $\rho$ centered at the point $z$, and

$$
I^{\mathscr{D}}=\left\{z_{1}, z_{2}, \ldots, z_{n}\right\} .
$$

If we let $\rho$ tend to zero at an appropriate rate this choice of recognition algorithm is consistent.

THEOREM 5.1. The set $I^{*}$ defined in (5.2) defines a consistent recognition algorithm for any $I \in \mathscr{T}$ (as defined in $\S 1$ ) if $\mathscr{D}=\mathscr{D} 1$ and

$$
\rho \rightarrow 0, \quad n \rho^{2} \rightarrow \infty .
$$

Proof. Writing $I^{*}(z), I(z)$ for the indicator functions of $I$ and $I^{*}$ respectively, the error can be written as

$$
E e\left(I, I^{*}\right)=\int_{z \in I}\left[1-E I^{*}(z)\right] m(d z)+\int_{z \notin I} E I^{*}(z) m(d z) .
$$

Introduce the sets $I_{\rho}^{\text {in }}, I_{\rho}^{\text {out }}$ (see Figure 5.1) as

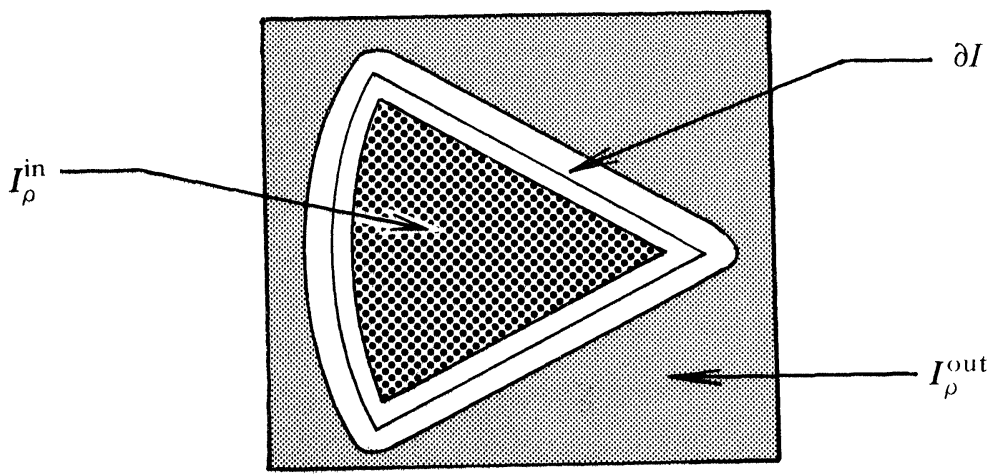

FIGURE 5.1

$$
\begin{aligned}
& I_{\rho}^{\text {in }}=\{z \mid z \in I \text { and } \operatorname{dist}(z, \partial I) \geqq \rho\}, \\
& I_{\rho}^{\text {out }}=\{z \mid z \notin I \text { and } \operatorname{dist}(z, \partial I) \geqq \rho\} .
\end{aligned}
$$

For $z \in I_{\rho}^{\text {in }}$, we have

$$
E I^{*}(z)=1-\left(1-\pi \rho^{2} / m(I)\right)^{n} \rightarrow 1
$$

because of (5.4). Similarly, for $z \in I_{\rho}^{\text {out }}$ we have

$$
E I^{*}(z) \equiv 0 .
$$

Combined with $m\left(\left(\sim I_{\rho}^{\text {out }}\right) \cap\left(\sim I_{\rho}^{\text {in }}\right)\right) \rightarrow 0$ which also follows from (5.4), the equations (5.7), (5.8) prove the assertion. 
If $\mathscr{D}=\mathscr{D} 3$ another approach is needed. Say that

$$
\lambda_{1}=\mu_{1} \lambda, \quad \lambda_{2}=\mu_{2} \lambda,
$$

with $\mu_{1}>\mu_{2}, n \rightarrow \infty$, and introduce

$$
I^{*}=\left\{z \mid N_{\rho}(z) \geqq t\right\}
$$

where $N_{\rho}(z)$ is the number of $z_{i}$ falling inside $C_{\rho}(z)$. We then have consistency:

THEOREM 5.2. The set (5.10) leads to consistent recognition for any $I \in \mathscr{T}, \mathscr{D}=\mathscr{D} 3$, if

$$
t=\left(\left(\mu_{1}+\mu_{2}\right) / 2\right) \pi \rho^{2} \lambda, \quad \rho \rightarrow 0, \quad \rho^{2} \lambda \rightarrow+\infty .
$$

The proof parallels the earlier one.

These two theorems guarantee that $I^{*}$ is close to $I$ in measure if $n$ or $\lambda$ is large. It should be kept in mind, though, that the speed of convergence of these recognition algorithms may be low. That this is indeed so will be seen at the end of this section. There is nothing surprising in this: the algorithms are quite general and do not assume any particular algebraic structure. Low efficiency is the price we have to pay for general validity.

This raises the question whether one could not build the recognition algorithm directly on the image algebra and the knowledge of $\mathscr{D}$. The following three examples clarify this approach.

The first one is very simple: $I$ is just $g_{0} C$, where $g_{0}$ is an unknown translation and $C$ is a circle of given radius $R$ (see Figure 5.2). The inner set in

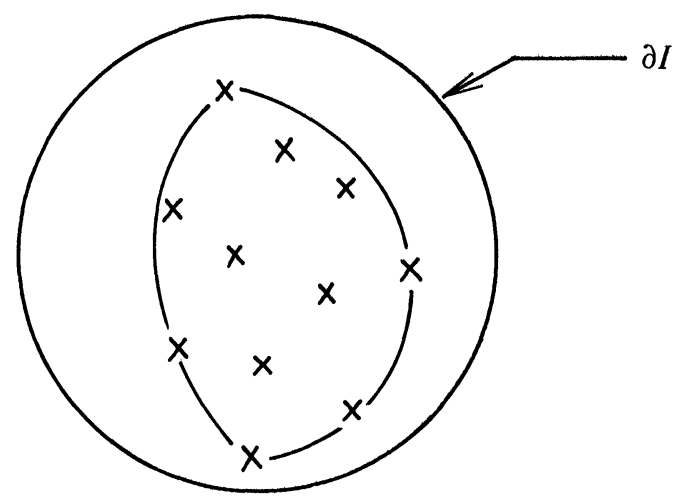

FIGURE 5.2

this figure bounded by circular arcs with radius $R$ will be denoted $I^{f}$ and has been constructed as the intersection of all $g C$ containing the deformed image $I^{\mathscr{D}}$ : 


$$
I^{f}=\bigcap_{(g C)>I^{g^{g}}} g C .
$$

It is clear that $I^{f} \subset I$ and, at least intuitively, that the expected error of $I^{f}$ tends to zero as $n$ increases. Note that $I^{f}$ is the feature closure of $I^{\mathscr{D}}$ (see Grenander (1968)).

The next example is $I=\left(g_{1} S_{1}\right) \cap\left(\sim g_{2} S_{2}\right)$ from Figure 1.1e. Starting from $I^{\mathscr{D}}$ we now form the intersection of all $g_{1} S_{1}$ and of $\sim g_{2} S_{2}$ containing $I^{\mathscr{D}}$. We get the inner set of Figure 5.3 and it is intuitively clear that this $I^{f}$ is also consistent. Note that it is the feature closure of $I^{\mathscr{D}}$ using the features $g S_{1}$ and $\sim g S_{2}$.

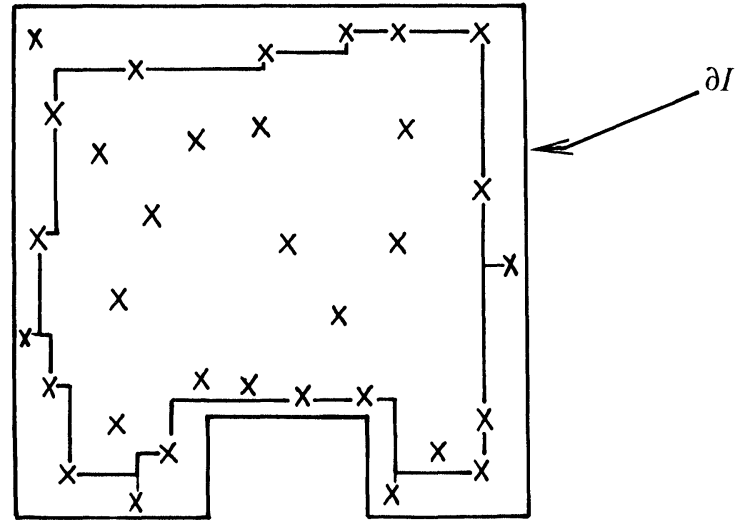

FiguRe 5.3

In the third example we use the disjunctive image of Figure 1.1d and form $I^{f}$ as the intersection of all sets with the syntax $\left(g_{1} C\right) \cup\left(g_{2} C\right)$. The resulting set will be bounded by circular arcs and it is clear again that consistency holds. $I^{f}$ is now a multiple feature closure of $I^{\mathscr{D}}$.

To treat a more general image algebra we shall need a weak additional condition. If $z^{\prime}, z^{\prime \prime}$ are two interior points close to each other and to the boundary of $I$ we shall denote by $a\left(z^{\prime}, z^{\prime \prime}\right)$ the inward-oriented area of $I$ excluded by intersection in (5.15) and bounded by the chord from $z^{\prime}$ to $z^{\prime \prime}$. We shall assume that

$$
\text { (A): } \quad a\left(z^{\prime}, z^{\prime \prime}\right)=O\left(\left\|z^{\prime}-z^{\prime \prime}\right\|^{2}\right) .
$$

Condition (A) is satisfied if the curvature is piecewise continuous and bounded and if the (finite number of) corners have positive opening angle.

THEOREM 5.3. Consider the pure image

$$
I=B\left(s_{1}, s_{2}, \ldots, s_{k}\right)
$$


where $B$ is a given Boolean function. Let

$$
I^{f}=\bigcap B\left(s_{\alpha_{1}}, s_{\alpha_{2}}, \ldots, s_{\alpha_{k}}\right)
$$

where the intersection is taken over all $\left(s_{\alpha_{1}}, s_{\alpha_{2}}, \ldots, s_{\alpha_{k}}\right)$ such that $B\left(s_{\alpha_{1}}, s_{\alpha_{2}}, \ldots, s_{\alpha_{k}}\right)$ contains $I^{\mathscr{D}}$ and $s_{\alpha_{1}}, s_{\alpha_{2}}, \ldots, s_{\alpha_{k}}$ are generated from the given set of prototypes via similarity transformations. 4ssuming that Condition (A) holds, $I^{f}$ represents a consistent recognition algorithm.

Proof. We have $I^{f} \subset I$ since one of the factors of the intersection in (5.15) is $I$ itself. Now consider $\mathscr{J}_{\rho}=I-I_{\rho}^{\text {in }}$ for small values of $\rho$. Note that $\partial I$ consists of a finite number of analytic arcs, say of total length $L$. As $n$ increases $\mathscr{J}_{\rho}$ will be more and more densely filled with points $z_{v} \in I^{\mathscr{D}}$. Let us order them with respect to curvilinear coordinates following each other in the direction of the arcs of $\partial I$. Consider two such points $z^{\prime}$ and $z^{\prime \prime}$ close to each other. The area of $I$ will not be completely covered by $I^{f}$. Neglecting the set $\mathscr{J}_{\rho}$, which tends to zero asymptotically, $I^{f}$ may miss some areas of $I$ due to inward indentations of $I^{f}$. The contribution due to the area associated with $\left(z^{\prime}, z^{\prime \prime}\right)$ is bounded by $a\left(z^{\prime}, z^{\prime \prime}\right)$ as in (5.17). The entire contribution is then bounded by

$$
\sum a\left(z_{v}, z_{v+1}\right)=O\left(\sum\left\|z_{v}-z_{v+1}\right\|^{2}\right)
$$

summed over the $v$ 's belonging to $z_{v} \in \mathscr{J}_{\rho}$. But the expected value of the right-hand side of (5.20) tends to zero as $n$ tends to infinity, and this completes the proof.

It would be interesting to find out how much better feature closure works as recognition algorithm compared to the general algorithm of Theorem 5.1. In other words, is it true that restoration algorithms that preserve structure are superior to general ones? This will be studied in a forthcoming paper (Grenander and Lavin (1973)).

ACKnowledgement. I am grateful to Professor A. C. Pipkin for pointing out an error in (2.9) in an earlier version of the manuscript.

\section{REFERENCES}

L. Bass and S. Schubert (1967), On finding the disc of minimum radius containing a given set of points, Math. of Comp. v. 21, 712-714.

W. Blaschke (1955), Vorlesungen über Integral-geometrie, Deutscher Verlag der Wissenschaften, Berlin.

S. Carlsson and U. Grenander (1967), Statistical approximation of plane convex sets, Skand. Aktuarietidskrift 3-4, 113-127. MR 37 \#6005.

R. Deltheil (1920), Sur la théorie des probabilités géometriques, Université Paris, Toulouse.

U. Grenander (1968), A feature logic for clusters, Report no. 1, Pattern Analysis, Center for Computer and Information Sciences, Brown University, Providence, R.I.

(1970), A unified approach to pattern analysis, Advances in Computers, vol. 10, edited by W. Freiberger. 
U. Grenander and P. Lavin (1973), Structure-preserving restoration of images, Report no. 7, Pattern Analysis, Center for Computer and Information Sciences, Brown University, Providence, R.I.

R. E. Kowalczyk (1972), Stochastic elastic deformation models for pattern analysis, Ph.D. Thesis, Division of Applied Mathematics, Brown University, Providence, R.I.

A. Rényi and R. Sulanke (1963/64), Ueber die Konvexe Hülle von $n$ zufällig gewählten Punkten, Z. Wahrscheinlichkeitstheorie und Verw Gebiete 2 75-84; ibid. 3 138-147. MR 27 \#6190; 29 \#6392.

L. A. Santaló (1936), Integralgeometrie, Hermann, Paris.

Current address: Division of Applied Mathematics, Brown University, Providence, Rhode Island 02912 